\title{
Publikationsunterstützung an der Universität Graz: Themen, Zielgruppen, Formate
}

https://doi.org/10.1515/bfp-2018-0011

Zusammenfassung: Die „Publikationsservices“ an der Universitätsbibliothek Graz haben in Kooperation mit unterschiedlichen Verwaltungseinheiten sowie mit den sechs Fakultäten der Universität ein breites Serviceportfolio zur Publikationsberatung entwickelt. Dieser Beitrag schildert den Aufbau und die Weiterentwicklung eines umfassenden Informations-, Beratungs- und Veranstaltungsangebots zum wissenschaftlichen Publizieren und zieht eine erste Zwischenbilanz.

Schlüsselwörter: Publikationsunterstützung; Bibliothek; wissenschaftliches Publizieren

\section{Publication Support at the University of Graz: Topics, Target Groups, Formats}

Abstract: The Publication Services, located at the University Library at the University of Graz, have developed a wide range of services with regard to scholarly communications and publishing by cooperating with various administrative units and with the University's faculties. This paper will delineate how its service portfolio has been created and refined by entailing provision of information resources, consultancy services, workshops and lectures.

Keywords: Publishing support; library; scholarly publishing

\section{Entstehung und Struktur der Stabsstelle Publikationsservices}

In den vergangenen Jahren haben zahlreiche wissenschaftliche Bibliotheken Infrastrukturen und Beratungsangebote zu unterschiedlichen Aspekten des wissenschaftlichen Publizierens auf- und ausgebaut. Während jedoch im anglo-amerikanischen Raum die Koordination und Verknüpfung publikationsunterstützender Angebote weit verbreitet ist, sind im deutschen Sprachraum zusam-

*Kontaktpersonen: Mag. Christian Kaier, christian.kaier@uni-graz.at MMag. Karin Lackner, karin.lackner@uni-graz.at menhängende und umfassende Serviceportfolios in diesem Bereich erst im Aufbau. ${ }^{1}$

An der Karl-Franzens-Universität Graz wurde im Entwicklungsplan 2013-2018 ein strategisches Projekt zum Aufbau publikationsunterstützender Angebote festgelegt und im August 2015 an der Universitätsbibliothek die Stabsstelle „Publikationsservices“, bestehend aus je zwei Mitgliedern der Abteilung Zeitschriften und Datenbanken sowie der Abteilung Informationsdienste, eingerichtet. Zunächst waren die Publikationsservices lediglich als Koordinierungsstelle der einzelnen, bereits bestehenden Bibliotheksservices im Bereich des wissenschaftlichen $\mathrm{Pu}$ blizierens gedacht. Basierend auf Publikationsinfrastrukturen wie dem institutionellen Repositorium uni $=$ pub für Zweitveröffentlichungen, Hochschulschriften und GoldOpen-Access-Journals und dem Universitätsverlag sowie der Verwaltung von Open-Access-Fördermöglichkeiten waren von Beginn an auch Beratungsangebote etwa zu Open Access und Bibliometrie Teil des Angebots der Stabsstelle. Es zeigte sich jedoch sehr bald, dass die Abstimmung mit anderen Abteilungen der Universität und deren Expertise wesentlich für die Arbeit der Stabsstelle sein würde. Beratung und Veranstaltungen zum wissenschaftlichen Publizieren werden innerhalb der Universität von verschiedenen Stellen angeboten: Im Rahmen der internen Weiterbildung halten etwa Gastvortragende Workshops zu verschiedenen Aspekten des Publikationsprozesses ab, einzelne Fakultäten bieten spezialisierte Veranstaltungen zum Publizieren in den jeweiligen Disziplinen und das DocService, das den wissenschaftlichen Nachwuchs am Beginn seiner wissenschaftlichen Karriere unterstützt, berät Doktoranden auch zu Publikationsthemen.

Daher erschien es dem Team der Publikationsservices wichtig, den Kontakt mit diesen und weiteren Abteilungen zu vertiefen. Als Kooperationspartner konnten bislang gewonnen werden: das DocService, die Rechts- und Organisationsabteilung, die unter anderem Rechtsberatung zu Urheberrecht, Bildrecht und Verlagsverträgen anbietet, das Leistungs- und Qualitätsmanagement, mit dem die Bibliothek im Bereich Bibliometrie und Performance-Mes-

1 Weitere Informationen zum neuen Aufgabenfeld der Publikationsberatung siehe Kaier (2017). 
sung schon zuvor zusammengearbeitet hatte, sowie das Forschungsmanagement und -service, mit dem hinsichtlich Förderberatung und Verwertung von Forschungsergebnissen ebenfalls Kontakt bestanden hatte.

Der offizielle Startschuss der „Publikationsservices“ als Kooperationsplattform zwischen der Bibliothek und den genannten Verwaltungseinheiten der Universität erfolgte im April 2016. ${ }^{2}$

\section{Themen}

Die Basis des Themenportfolios bildeten zunächst jene Bereiche, zu denen es bereits vor Gründung der Stabsstelle Beratungsangebote an der Universitätsbibliothek gegeben hatte. Dazu zählten Open Access und Zweitveröffentlichungsrechte, Open-Access-Abkommen mit Verlagen, Bibliometrie und das Publizieren im Universitätsverlag und auf dem institutionellen Repositorium. Themenbereiche wie Literaturrecherche und Quellenbewertung, die zum Schreibprozess und damit indirekt zum Publikationsprozess zählen, wurden bewusst ausgeklammert, da diese Themen von der an der Universitätsbibliothek angesiedelten Stabsstelle Informationskompetenz abgedeckt werden.

Mit diesem thematisch zunächst noch recht kleinen Themenportfolio wurde ein erster Entwurf für ein Beratungs- und Servicekonzept erstellt und den Kooperationspartnern präsentiert. Bei den Gesprächen stellte sich rasch heraus, dass das Themenspektrum auf weitere Aspekte ausgedehnt werden sollte, zu denen es an der Universität noch kaum oder gar keine Angebote gab. Aus Sicht der Publikationsservices ergaben sich noch weitere Themen wie Forschungsdatenmanagement und die Erstellung von Data Management Plans. Um nicht am tatsächlichen Bedarf vorbeizuplanen, wurden im Lauf des Jahres 2016 die Vizedekane für Forschung aller sechs Fakultäten ${ }^{3}$ der Universität kontaktiert. Für die Gespräche wurde ein Fragebogen entwickelt, der Fragen zu den bestehenden Beratungsund Publikationsangeboten, zu Erfahrungen mit Open Access und Forschungsdatenmanagement und zu Publikationskosten und Förderangeboten im jeweiligen Fachgebiet enthielt. ${ }^{4}$ In den Gesprächen wurde außerdem geklärt,

2 Weitere Informationen zum Aufbau der Stabsstelle Publikationsservices an der Universität Graz siehe Lackner und Ginther (2017).

3 Katholische Theologie, Rechtswissenschaften, Sozial- und Wirtschaftswissenschaften, Geisteswissenschaften, Naturwissenschaften, Umwelt-, Regional- und Bildungswissenschaften.

4 Einige Beispiele: Welche Aspekte des wissenschaftlichen Publizierens sind an Ihrer Fakultät von besonderem Interesse? Wie können welche Themen für die Wissenschaftler bzw. für die jeweilige Fakultät besonders interessant und wichtig waren, und es wurde ermittelt, ob ein Wunsch nach entsprechenden neuen Angeboten besteht oder der diesbezügliche Informationsbedarf möglicherweise ohnedies über andere Kanäle gedeckt wird. Dabei ergaben sich einige zusätzliche Themenbereiche, die nach Auswertung aller Gespräche in das Themenportfolio der Publikationsservices aufgenommen wurden, beispielsweise der häufig genannte Wunsch nach mehr Information bezüglich Urheberrecht und Bildrecht. Insgesamt ergaben sich aus den Gesprächen drei thematische Schwerpunkte:

- Prozess des Publizierens

- Publizieren an der Universität Graz

- Verwertung und Sichtbarmachung von Forschungsergebnissen

Die ursprüngliche Themenliste wurde auf Basis der in den Gesprächen genannten Themen auf insgesamt 14 Bereiche erweitert und den thematischen Schwerpunkten zugeordnet:

Prozess des Publizierens

- Grundlagen des Publizierens

- Prozess des Publizierens in Journals

- Open Access

- Creative-Commons-Lizenzen

- Urheberrecht und Bildrecht

- Verlagsverträge

- Open-Access-Policies des Fonds zur Förderung der wissenschaftlichen Forschung (FWF) und anderer Förderorganisationen

- Forschungsdatenmanagement und Data Management Plans

Publizieren an der Universität Graz

- Publizieren an der Universität print und digital

- Publikationsserver uni $=$ pub

- Open-Access-Abkommen

Verwertung und Sichtbarmachung von Forschungsergebnissen

- Bibliometrie

- Die Sichtbarkeit der eigenen Forschung erhöhen

die Publikationsservices das Publizieren an Ihrer Fakultät unterstützen? Wie sind die Erfahrungen mit Open Access in Ihrem Fachbereich? Welche Bedeutung haben bibliometrische Daten in Ihrem Bereich (qualitativ und quantitativ)? Wie können wir Sie im Bereich Forschungsdatenmanagement unterstützen? Welche Formen der Publikationsförderung gibt es an Ihrer Fakultät und welche (externen) Fördergeber sind in Ihrem Fachbereich relevant? 
- Aktuelle Trends in der wissenschaftlichen Kommunikation

Im ersten Halbjahr 2017 wurde eine zweite Gesprächsrunde mit den sechs Vizedekanen für Forschung geführt und ein Feedback zum Themenportfolio eingeholt. Die Ergebnisse zeigen, dass die Themen den Wünschen und Erwartungen bereits weitgehend entsprechen, jedoch wurden auch neue Themen zur Sprache gebracht. Dazu zählten beispielsweise der Wunsch nach Präsentationsseminaren - insbesondere auf Englisch - sowie die Aufnahme des Themas „Predatory Publishing“ ins Themenportfolio. Hier zeigte sich, dass die Abgrenzung zu anderen Verwaltungseinheiten und deren Angeboten zunehmend wichtiger wird: Präsentationsseminare werden regelmäßig von der Personalentwicklung angeboten. Das Thema „Predatory Publishing“ wurde ausgearbeitet und das Themenportfolio damit auf fünfzehn Themen erweitert.

\section{Zielgruppen}

Für den Aufbau des Serviceportfolios wurde des Weiteren eine Zielgruppenanalyse durchgeführt. Dabei wurden drei Zielgruppen und deren Bedarfe identifiziert:

- Doktoranden und Nachwuchswissenschaftler, die vor ihrer ersten Publikation stehen oder bereits einige wenige Papers veröffentlicht haben, aber noch großen Informationsbedarf auch bei grundlegenden Fragen zum Publizieren haben.

- Wissenschaftler, die schon viel Erfahrung im Publizieren haben, aber an Informationen zu einigen spezifischen Fragen bzw. neu aufkommenden Themen interessiert sind.

- Neu an die Universität gekommene (Gast-)Dozenten bzw. (Gast-)Professoren, denen Best-Practice-Beispiele sowie Informationen zur Publikationsunterstützung durch die Universität Graz - beispielsweise über das Repositorium oder den Universitätsverlag - zur Verfügung gestellt werden sollen.

Zur selben Zeit entschied sich eine junge Kollegin, im Rahmen ihres Praktikums an der Universitätsbibliothek ein strategisches Marketing-Konzept für die Publikationsservices $\mathrm{zu}$ erarbeiten. Aufgrund ihrer betriebswirtschaftlichen Ausbildung entstand ein wertvoller Beitrag zur Weiterentwicklung der Stabsstelle: Unter anderem identifiziert das interne Papier verschiedene Kategorien von Kontakten und Ansprechpartnern für den Aufbau eines Netzwerks an der Universität. Die Kommunikation mit den Zielgruppen erfolgt unter anderem über ein informelles Netz sogenann- ter Advocates, Ambassadors, Consultants und Affiliates, ${ }^{5}$ die selbst als Wissenschaftler tätig sind, Informationen teilen und die Angebote der Publikationsservices weiterempfehlen.

Im Zuge der Gespräche mit Fakultätsleitungen und Kooperationspartnern zeigte sich, dass bestimmte Zielgruppen verstärkt angesprochen werden sollten, darunter Studierende und sog. „Early Career Researchers“. Die Wissensvermittlung an Studierende ist dabei als Investition in die Zukunft zu sehen, da einige von ihnen in wenigen Jahren selbst wissenschaftlich tätig sein werden. Wissenschaftler verfügen zu Beginn ihrer Laufbahn oft noch nicht über breite Publikationserfahrung und sind insgesamt entsprechenden Angeboten gegenüber aufgeschlossener als etablierte Wissenschaftler. Im Laufe von Gesprächen stellt sich aber des Öfteren heraus, dass eine auf Publikationsthemen spezialisierte Einrichtung wie die Publikationsservices bei Themen wie Open Access, Bibliometrie, Academic Social Media und ORCID fallweise durchaus neue und relevante Informationen anzubieten haben. Umgekehrt profitieren die Publikationsservices vom Kontakt mit etablierten Wissenschaftlern, etwa durch deren Erfahrungen zur Publikationspraxis in den jeweiligen Disziplinen und zu Erfahrungen mit Predatory Publishing.

Das ambitionierte Ziel der Publikationsservices ist es, ein thematisch breites Angebot an eine diverse, mit unterschiedlichem Vorwissen ausgestattete Gruppe von Wissenschaftlern zu vermitteln und damit die Publikationstätigkeit an der Universität zu unterstützen. Im Unterschied dazu sind vergleichbare Angebote an anderen Universitäten bzw. Bibliotheken teilweise als Weiterbildung für Bibliothekare konzipiert oder umfassen meist „nur“ Themen, die sich mit dem Open-Access-Publizieren befassen. In der Regel treten dabei Bibliotheken als alleinige Anbieter entsprechender Informationen auf, während an der Universität Graz eine gemeinsame Anlaufstelle unterschiedlicher Einrichtungen etabliert wurde. Im Unterschied zu den im angloamerikanischen Raum verbreiteten „Scholarly Communications Offices“ fehlt an der Universität Graz jedoch (zumindest derzeit) eine institutionelle Verankerung über die Bibliothek hinaus.

\section{Formate}

Basierend auf dem ursprünglichen Beratungs- und Servicekonzept, der Zielgruppenanalyse, den Gesprächen mit

5 Vgl. Mathews (2009). 
den Vizedekanen und dem ausgearbeiteten Themenportfolio wurde ein Informations- und Beratungsangebot entwickelt, das sowohl den unterschiedlichen Bedürfnissen der oben erwähnten Zielgruppen als auch individuellen Vorlieben bezüglich Informationsbeschaffung Rechnung trägt:

- Informationsmaterial: Die wichtigsten Informationen $\mathrm{zu}$ den verschiedenen Themenbereichen werden sowohl über die Homepage der Publikationsservices als auch über Info-Folder in Form von kurzen, übersichtlichen Texten zur Verfügung gestellt. Auf der Homepage finden sich darüber hinaus Links zu weiterführenden Seiten sowie Unterlagen und Materialien zum Download.

- Schriftliche, telefonische und persönliche Beratung: Die Publikationsservices stehen sowohl über eine zentrale E-Mail-Adresse als auch telefonisch für Anfragen zur Verfügung und bieten auch persönliche Beratung nach vorheriger Terminvereinbarung an.

- Buchbare Kurzworkshops: Zu allen oben genannten Themenbereichen wurden 30- bis 45-minütige Vorträge erarbeitet, die auf Anfrage in Lehrveranstaltungen wie beispielsweise Doktorandenseminare, Doktorandenkolloquien oder sonstige (Instituts-)Veranstaltungen modulartig eingebaut werden können. Die Kurzworkshops können auf Wunsch auch kombiniert werden, so besteht beispielsweise die Möglichkeit, eine 1,5-stündige Lehrveranstaltungseinheit aus zwei bis drei Kurzworkshops zusammenzustellen und komplett von den Publikationsservices gestalten zu lassen. ${ }^{6}$

- Regelmäßige (Gast-)Vorträge und Workshops: Die Publikationsservices organisieren jedes Semester einige Veranstaltungen $\mathrm{zu}$ wechselnden Themen aus dem Themenportfolio, die teils von den Mitgliedern und/ oder Kooperationspartnern der Publikationsservices, teils von eingeladenen Gastvortragenden und externen Experten abgehalten werden. Diese Veranstaltungen finden zu fixen Terminen statt und stehen allen Universitätsangehörigen offen. ${ }^{7}$

\footnotetext{
6 Die Inhalte der Kurzworkshops, basierend auf dem aktuellen Themenportfolio, können auf der Homepage der Publikationsservices (ub.uni-graz.at/publikationsservices) unter dem Menüpunkt „Veranstaltungen“ eingesehen werden. Neben einer Kurzbeschreibung des jeweiligen Themas bzw. Workshops werden gegebenenfalls weiterführende Links sowie ein Link zu passender Literatur im Online-Katalog der Universitätsbibliothek Graz, uni=kat, angeboten.

7 Die Vorträge und Workshops des aktuellen Semesters sind im Campussystem der Universität Graz, UNIGRAZonline (https://online. uni-graz.at), im Kursangebot der Universitätsbibliothek zu finden.
}

Alle Angebote der Publikationsservices können von den Angehörigen der Universität kostenfrei genutzt werden. Je nach Verfügbarkeit freier Plätze können die Vorträge und Workshops darüber hinaus auch von externen Interessierten kostenfrei besucht werden.

\section{Bisherige Veranstaltungen}

Die erste Veranstaltung der Publikationsservices zum Thema Bild- und Urheberrecht fand im November 2016 statt. Aufgrund der unerwartet hohen Nachfrage (knapp 100 Teilnehmer) wurde die Veranstaltung in die Aula der Universität verlegt. Die Rückmeldungen der Teilnehmer waren ausgesprochen positiv, insbesondere die Möglichkeit, Informationen aus erster Hand über die Tätigkeiten von Verwertungsgesellschaften zu erhalten, sowie die zahlreichen Fallbeispiele, die die Vortragenden aus eigener Erfahrung berichteten, fanden großen Anklang.

Im Sommersemester 2017 organisierten die Publikationsservices mit je einer Veranstaltung zu Creative Commons, der Open Access Policy des Fonds für wissenschaftliche Forschung (FWF), sowie mit einem Workshop zum Publizieren mit Formatvorlagen drei weitere Veranstaltungen, zu denen Experten als Gastvortragende eingeladen wurden. Zusätzlich hielt das Team der Publikationsservices selbst einen Workshop zu den Grundlagen des Publizierens ab. Im Studienjahr 2016/2017 besuchten insgesamt etwa 200 Angehörige des wissenschaftlichen und administrativen Personals sowie auch zahlreiche Doktoranden die Veranstaltungen der Publikationsservices. Jede Veranstaltung wurde evaluiert und die Ergebnisse jeweils für die folgenden Veranstaltungen berücksichtigt.

Im Wintersemester 2017/2018 wurde ein Workshop zu Open Access sowie aufgrund der starken Nachfrage ein weiterer Workshop zum Thema Bild- und Urheberrechte angeboten. Zum ersten Mal organisierten die Publikationsservices außerdem mit dem „Tag des wissenschaftlichen Publizierens“ eine gemeinsame, ganztägige Veranstaltung in Zusammenarbeit mit ihren vier Kooperationspartnern (Rechts- und Organisationsabteilung, DocService, Leistungs- und Qualitätsmanagement, Forschungsmanagement und -service) sowie weiteren Servicestellen und Verwaltungseinheiten der Universität, darunter dem Schreibzentrum, der Abteilung Presse + Kommunikation sowie der Stabsstelle Informationskompetenz. Erfreulicherweise fand die Idee sowohl bei den Kooperationspartnern als auch den weiteren Stellen großes Interesse, sodass alle kontaktierten Personen sich mit einem halbstündigen Vortrag beteiligten. Die Themen reichten von der Themen- 
findung sowie Literaturrecherche und -verwaltung über Predatory Publishing und Bildrechte hin zu Forschungsdatenmanagement, Bibliometrie und Medien- und Öffentlichkeitsarbeit. Daneben war es auch ein ausdrückliches Ziel der Veranstaltung, die beteiligten Abteilungen und Servicestellen mit ihrem Beratungs- und Serviceangebot kurz vorzustellen. Um den Teilnehmern je nach Zielgruppe und Interessenschwerpunkt ein gezieltes Besuchen einzelner Themenblöcke bzw. Vorträge zu ermöglichen, wurde ein genaues, thematisch strukturiertes Tagesprogramm erstellt.

Trotz intensiver Bewerbung blieb die Teilnehmerzahl mit rund 30 Personen - darunter Studierende und Angehörige des wissenschaftlichen und administrativen Personals der Universität Graz sowie externe Personen - hinter den Erwartungen zurück. Dennoch zeigt die Teilnahme mehrerer externer Personen, teils sogar aus anderen Bundesländern, dass die behandelten Inhalte sowie auch die publikationsunterstützenden Maßnahmen und Serviceangebote an der Universität Graz über die Angehörigen der eigenen Universität hinaus auf Interesse stoßen. Auch die Evaluation der Veranstaltung fiel hervorragend aus. Ein weiterer positiver Effekt der Veranstaltung war ihr Beitrag zur weiteren Vernetzung der Publikationsservices mit allen an der Veranstaltung beteiligten Verwaltungseinheiten und Servicestellen. Ob und in welcher Form das aufwändige Format eines „Tages des wissenschaftlichen Publizierens“ im Jahr 2018 fortgesetzt wird, wird gemeinsam mit den Kooperationspartnern zu entscheiden sein.

\section{Erkenntnisse}

Die Vernetzung an der Universität und die Erstellung eines Serviceportfolios für die Publikationsberatung brachten dem Team der Publikationsservices wertvolle Einsichten:

- Es entstand und entsteht ein größeres Bild des Research Life Cycle durch die Einbindung von Kollegen, die auf Forschungsunterstützung, Evaluation, Rechtsfragen und andere angrenzende Themen spezialisiert sind.

- Die Vernetzung mit anderen Verwaltungseinheiten bringt einen Mehrwert durch die bessere Abstimmung der unterschiedlichen Angebote und die leichtere Identifikation thematischer Lücken. Vernetzung bedeutet dabei insbesondere auch das Teilen von Informationen.

- Know-How entsteht durch den Austausch mit Wissenschaftlern in beiderlei Richtung, wertvolle Hinweise erweitern Umfang und Praxisbezug des eigenen Wissensstandes.
- Die Publikationskulturen unterschiedlicher Disziplinen unterscheiden sich so fundamental, dass eine fachliche Durchdringung kaum möglich scheint. Andererseits wird die Vermittlung aktueller Entwicklungen von Wissenschaftlern durchaus geschätzt.

- Die Weitergabe von Informationen ist dann besonders erfolgreich, wenn sie nicht in Form von Lobbying mit konkreten Zielvorstellungen erfolgt (z. B. klare Darstellung von Vorteilen, aber auch Bedenken gegenüber Open Access).

- Vertrauen schaffen und Kontakt halten mit Wissenschaftlern und den Serviceeinheiten ist eine zentrale Aufgabe der Publikationsservices. Vertrauens- und Kontaktpersonen („Ambassadors“ etc.) an den Fakultäten sind enorm wichtig für die Etablierung und Weiterentwicklung der Angebote.

- Der erste „Tag des wissenschaftlichen Publizierens“ hat aber auch gezeigt, dass der Aufbau neuer Services Zeit und Überzeugungsarbeit benötigt. Viele Wissenschaftler haben die Publikationsservices noch immer nicht wahrgenommen oder trauen ihnen nicht $\mathrm{zu}$, nützliches „neues“ Wissen aus einem Kernbereich ihrer Arbeit anbieten zu können.

\section{Fazit und Perspektiven}

Die Publikationsservices haben Gastvorträge in Lehrveranstaltungen abgehalten, im Bereich der Personalentwicklung Workshops sowohl auf Deutsch als auch auf Englisch veranstaltet - auch hier waren die Themen Open Access, Sichtbarkeit, Rechtsfragen und Forschungsdatenmanagement besonders gefragt - und eine Veranstaltung mit Habilitanden unterschiedlicher Fachbereiche abgehalten. Damit konnten Studierende, Doktoranden und Wissenschaftler mit ganz unterschiedlichem Hintergrund und unterschiedlichem Umfang an Publikationserfahrung erreicht werden. Dazu kommen persönliche Gespräche zu Spezialthemen wie unter anderem Predatory Publishing, Bibliometrie und Zweitveröffentlichungsrechten.

Die oben beschriebenen Angebote der Publikationsservices werden laufend evaluiert. Aus dem bisherigen positiven Feedback lässt sich erkennen, dass Art und Inhalt der Angebote geschätzt und als hilfreich empfunden werden. Darüber hinaus können nach den ersten beiden Jahren des Aufbaus der Publikationsservices folgende Schlüsse gezogen und Vorhaben skizziert werden:

- $\quad$ Nicht alle Themenbereiche können von der Bibliothek allein abgedeckt werden, mitunter ist eine Kooperation mit spezialisierten Partnern sinnvoll. 
- Seitens der Kooperationspartner werden die Initiative der Bibliothek und der breite und oftmals informelle Austausch geschätzt. Die Bibliothek muss diesen Austausch aber weiterhin aktiv fördern und organisieren.

- Eine Verankerung der Publikationsservices im Organigramm der Universität ist bis dato nicht erfolgt, wäre aber wünschenswert, um die Kooperationen langfristig abzusichern.

- Ein weiterer Ausbau der Kooperationen scheint sinnvoll, da mit relevanten Einrichtungen wie Presse + Kommunikation, Doctoral Academy, Uni-IT, Welcome Center, Austrian Center for Digital Humanities oder Akademie für neue Medien zwar Gespräche geführt wurden, aber noch keine Kooperation vereinbart wurde. Eine solche Ausweitung müsste allerdings schrittweise erfolgen, um die Kapazitäten des Teams nicht zu überfordern.

- Wie die bisherigen Veranstaltungen gezeigt haben, sind Workshops zu bild- und urheberrechtlichen Themen besonders gefragt und sollen auch in Zukunft regelmäßig stattfinden. Darüber hinaus ist eine Ausweitung des Serviceangebots geplant, zunächst etwa um die Themen Wissenschaftliche Integrität und Gute wissenschaftliche Praxis.

- Weiterhin und dauerhaft ist es wichtig, Entwicklungen im Bereich der wissenschaftlichen Kommunikation und des wissenschaftlichen Publizierens zu verfolgen und zu vermitteln.

- Gemeinsame Veranstaltungen wie der „Tag des wissenschaftlichen Publizierens" fördern die Zusammenarbeit der verschiedenen Partner und bieten die Gelegenheit, die zahlreichen Aspekte des Themas „Wissenschaftliches Publizieren“ darzustellen. In welchem Format und Umfang eine derartige Veranstaltung in Zukunft angeboten werden soll, wird derzeit diskutiert. Darüber hinaus haben sich Kurzworkshops und Beratungsangebote als Formate bestens bewährt.

\section{Literaturverzeichnis}

Kaier, Christian (2017): Publikationsunterstützung: Die Bibliothek ist nicht genug: Kooperation als wesentlicher Erfolgsfaktor für Publikationsservices von Bibliotheken. In: Informationspraxis, 3 (1). Verfügbar unter http://dx.doi.org/10.11588/ip.2017.1.35225.

Lackner, Karin; Ginther, Clara (2017): Unterstützung des wissenschaftlichen Publizierens als Aufgabe einer wissenschaftlichen Bibliothek: Die Publikationsservices an der UB Graz. In: O-bib, (4), 154-63. Verfügbar unter: http://doi.org/10.5282/o-bib/201 7H4S145-154

Mathews, Brian Scott (2009): Marketing today's academic library: A bold new approach to communicating with students. Chicago, Ill.: American Library Association.

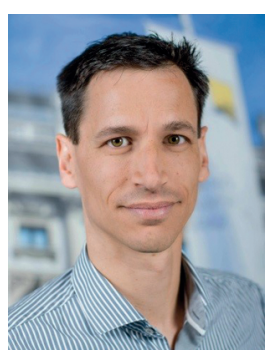

Mag. Christian Kaier

Universitätsbibliothek Graz

Open Access Office; Publikationsservices

Universitätsplatz $3 a$

A-8010 Graz

Österreich

christian.kaier@uni-graz.at

https://orcid.org/0000-0002-8750-6666

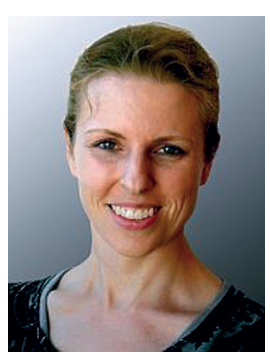

MMag. Karin Lackner

Universitätsbibliothek Graz

Open Access Office; Publikationsservices

Universitätsplatz $3 a$

A-8010 Graz

Österreich

karin.lackner@uni-graz.at

http://orcid.org/0000-0001-6096-1717 